\title{
Influence of Daidzein and Daidzein Metabolites on Lifespan and Stress Resistance in Caenorhabditis Elegans
}

\author{
Ye WANG ${ }^{1}$, Xiao-lei $\mathrm{LI}^{2}$, Cong-hui ZHANG ${ }^{3}$ and Xiu-ling WANG ${ }^{4, *}$ \\ ${ }^{1234}$ College of Life Sciences, Agricultural University of Hebei, Baoding, China \\ Ye Wang and Xiao-Lei Li contributed equally to this work.
}

Keywords: Daidzein, Daidzein metabolites, C. elegans, Lifespan, Stress resistance.

\begin{abstract}
Caenorhabditis elegans (C. elegans) was used as an animal model to study the influence of isoflavone daidzein and daidzein metabolites, including dihydrodaidzein (DHD), $O$-desmethylangolensin $(O$-Dma) and equol, on lifespan in $C$. elegans at the concentration of $0.1 \mathrm{mmol} / \mathrm{L}$. The results showed that equol significantly increased the lifespan of $C$. elegans; however, $O$-Dma significantly shortened the lifespan of $C$. elegans. No obvious difference in lifespan was observed when the worms were exposed to daidzein and DHD, respectively. Further study on stress resistance demonstrated that both the thermal and oxidative stress tolerance capacity of $C$. elegans was significantly enhanced after being exposed to equol.
\end{abstract}

\section{Introduction}

Isoflavones, which are mainly composed of daidzein and genistein are functional food factors recognized in soy. Studies have shown that soy isoflavones can exert multiple biological activities, such as antioxidant[1], anti-carcinogenesis[2], a reduced risk of cardiovascular diseases[3], and a potentially protective role for menopausal symptoms and osteoporosis [4]. However, more recently attention has shifted to isoflavone metabolites, which are the products of isoflavone metabolism via specific isoflavone biotransforming bacteria in the gut. Accumulative evidences suggest that daidzein metabolites including dihydrodaidzein (DHD), $O$-desmethylangolensin $(O$-Dma) and equol, are of stronger or wider bioactivities than their precursor isoflavones[5-7]. In our previous study, we have demonstrated that the microbial metabolites of daidzein, including $\mathrm{DHD}, O$-Dma and equol showed significantly stronger free-radical scavenging activity than that of daidzein in vitro[8].

Caenorhabditis elegans offers many advantages as a model for biological studies due to its simplicity, transparency, ease of cultivation, and completely sequenced genome[9]. In particular, its short life cycle makes it suitable for aging studies[10]. In the present study, we prepared the microbial metabolites of daidzein and investigated the anti-aging activity of daidzein and daidzein metabolites by using Caenorhabditis elegans as a model. In addition, both the thermal tolerance and oxidative stress tolerance were studied. Our results showed for the first time that equol not only significantly increased the lifespan of $C$. elegans but also significantly enhanced the resistance against both thermal and oxidative stress.

\section{Materials and Methods}

\section{Chemicals and Reagents}

Chemicals daidzein was purchased from Indofine (Somerville, NJ). Daidzein metabolites, including $\mathrm{DHD}[11]$., $O$-Dma[12]. and equol[13]., were prepared by using our previous 
microbial biotransformation methods. The enantiomeric excess (\% e.e.) of the biosynthesized DHD was zero and that of the biosynthesized $O$-Dma and equol was $88.3 \%$ and $100 \%$, respectively. In addition, equol is $100 \%(-)-S$-equol. FUDR (5-fluoro-2'-deoxyuridine) was bought from Sigma (98\% purity). Juglone (5-hydroxy-1, 4-naphthoquinone, Sigma-Aldrich, $97 \%$ purity), a reactive oxygen species-generating compound, was used to induce oxidative stress in worms.

\section{Worms and Escherichia Coli OP50}

Wild-type C. elegans N2 and Escherichia coli OP50 used in this study were kindly provided by Professor Chonglin Yang, Chinese Academy of Sciences, China. The worms were maintained and propagated on nematode growth medium (NGM) with standard techniques[14]. Strain E. coli OP50 was grown overnight in $\mathrm{LB}$ at $37^{\circ} \mathrm{C}$ in an incubator followed by being centrifuged $(4,000 \times \mathrm{g})$ for $10 \mathrm{~min}$ at $4^{\circ} \mathrm{C}$. The pellet was resuspended in $\mathrm{S}$ complete medium to an $\mathrm{OD}_{600}$ of approximately 0.90 and stored at $4^{\circ} \mathrm{C}$.

\section{Lifespan Analysis}

Lifespan assay were performed at $20^{\circ} \mathrm{C}$ in a LRH-250A biochemical incubator. Synchronized worms were transferred to 24-well treatment plates when they grew to L4 stage. The worms were then transferred to fresh well every 2 days. Dead worms were scored under NTB-4B stereoscopic microscope every day. A complete absence of swimming movement even after being touched with a small pipette tip was scored as death. Treatment plates were prepared using the reproductive suppressant FUDR $(50 \mu \mathrm{mol} / \mathrm{L})$ and the indicated final concentrations of daidzein and daidzein metabolites was $0.1 \mathrm{mmol} / \mathrm{L}$.

\section{Thermotolerance Assay}

Thermotolerance assay was performed with worms on adult day 2. Synchronized L4 worms exposed to $0.1 \mathrm{mmol} / \mathrm{L}$ of equol dispersed in live $E$. coli $\mathrm{OP} 50$ suspension were maintained at $20^{\circ} \mathrm{C}$ and shifted to $35^{\circ} \mathrm{C}$ when the thermotolerance assay started. The numbers of surviving and dead worms were scored every $1 \mathrm{~h}$.

\section{Oxidative Stress Assay}

Juglone sensitivity was assayed at $20^{\circ} \mathrm{C}$ using 1-day-old adults. At that time the worms exposed to $0.1 \mathrm{mmol} / \mathrm{L}$ of equol dispersed in live $E$. coli OP50 suspension were transferred onto 24-well plate with $300 \mu \mathrm{mol} / \mathrm{L}$ of juglone.

\section{Statistical Analyses}

Statistical analyses were carried out using the Kaplan-Meier method and differences in survival rates were tested using the Log-Rank test with 95\% confidence. Analysis of variance in group comparisons was performed by One-way ANOVA and significance of differences between groups were determined by a Tukey's multiple comparison tests. All statistical procedures were carried out in spss18.0 software.

\section{Results}

\section{Biosynthesis of Daidzein Metabolites}

Daidzein metabolites, including dihydrodaidzein (DHD), $\mathrm{O}$-desmethylangolensin ( $O$-dma) and equol, were prepared from the substrate daidzein by using microbial biotransformation method. 
Different daidzein metabolites were purified by semi-preparative high-performance liquid chromatography (HPLC). The purity of each biosynthesized DHD, $O$-Dma and equol is more than $99 \%$.
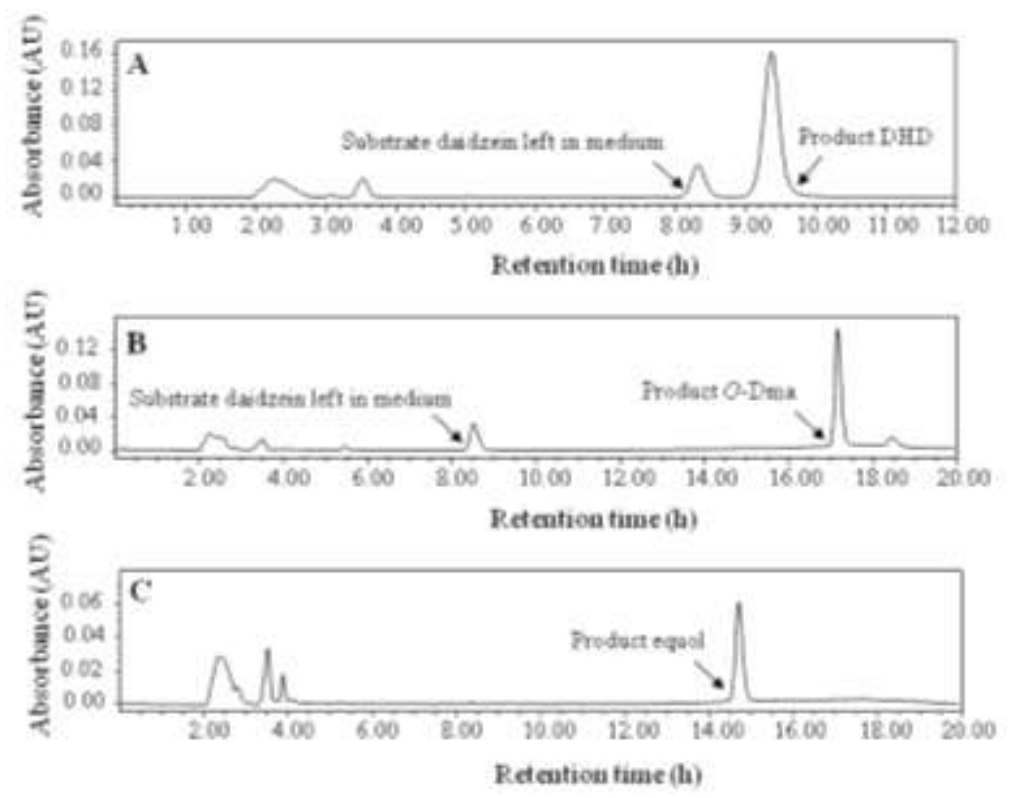

Fig.1 HPLC profiles for biosynthesis of daidzein metabolites

\section{Influence of Daidzein and Daidzein Metabolites on the Lifespan of C. elegans N2}

To determine whether daidzein and daidzein metabolites could affect the worm's lifespan, we presented daidzein and daidzein metabolites, including DHD, $O$-Dma and equol, to the worms (Fig.2). The mean lifespan (in mean number of days \pm standard error) of the control worms fed the standard laboratory food OP50 with $0.1 \mathrm{mmol} / \mathrm{L}$ of daidzein was $11.900 \pm 1.154 \mathrm{~d}$; that of the worms exposed to $0.1 \mathrm{mmol} / \mathrm{L}$ of DHD, $O$-Dma and equol were $11.100 \pm 1.350 \mathrm{~d}$, $9.467 \pm 0.859 \mathrm{~d}$ and $15.300 \pm 1.079 \mathrm{~d}$, respectively. Statistical analysis indicated that the mean lifespan of the worms exposed to $0.1 \mathrm{mmol} / \mathrm{L}$ of equol $(P<0.05)$ was significantly longer than those of the control worms exposed to $0.1 \mathrm{mmo} / \mathrm{L}$ of daidzein. However, on the contrary, the mean lifespan of the worms exposed to $0.1 \mathrm{mmol} / \mathrm{L}$ of $O$-Dma $(P<0.05)$ was significantly shortened. No obvious difference in lifespan was observed when the worms were exposed to the same concentration of DHD. In addition, we did not observe any change in body size when the worms exposed to daidzein and different daidzein metabolites in comparison with the control worms fed with OP50. 

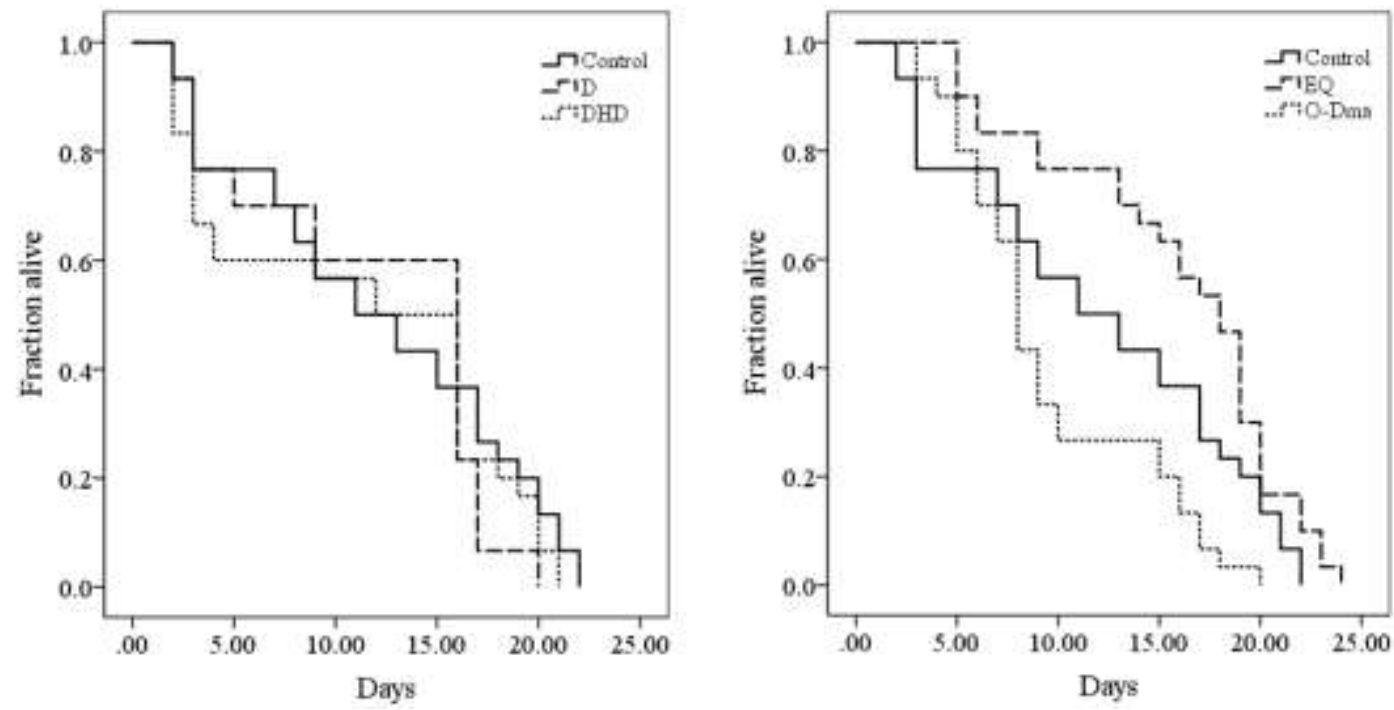

Fig.2 Effect of daidzein and daidzein metabolites on the lifespan of C. elegans

\section{Influence of Equol on Thermal Stress Resistance in C. elegans N2}

In order to investigate whether the increased longevity was associated with the improved survival capability under stress condition, we detected the thermal stress resistance of the worms exposed to $0.1 \mathrm{mmol} / \mathrm{L}$ (Fig.3). The results showed that the mean survival time for the controland treated worms at $35^{\circ} \mathrm{C}$ were $13.467 \pm 0.782 \mathrm{~h}$ and $15.933 \pm 0.835 \mathrm{~h}(P<0.05)$ respectively.

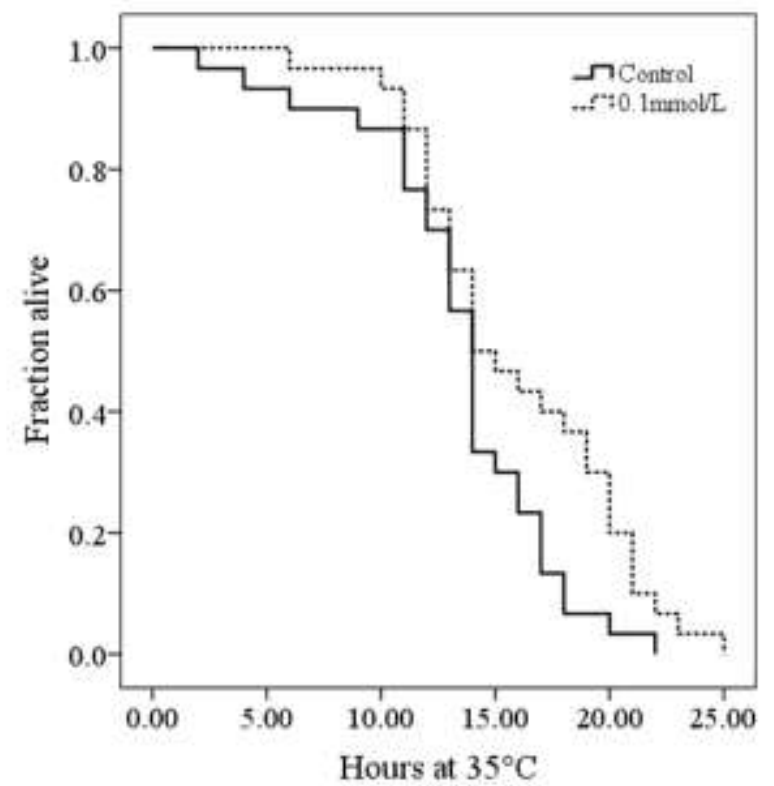

Fig.3 Effect of equol on resistance against thermal of C. elegans

\section{Influence of Equol on Oxidative Stress Resistance in C. elegans N2}

To evaluate the potential effect of equol on wild-type $C$. elegans $\mathrm{N} 2$ under oxidative stress, the worms were exposed to $300 \mu \mathrm{mol} / \mathrm{L}$ of juglone after being treated with $0.1 \mathrm{mmol} / \mathrm{L}$ of equol for 1 days (Fig.4). When the worms exposed to $0 \mathrm{mmol} / \mathrm{L}$ of equol (the control) and 0.1 $\mathrm{mmo} / \mathrm{L}$ of equol (the treatment) were continuously exposed to juglone for $10 \mathrm{~h}$, the average 
survival rate were $26.7 \%$ and $63.3 \%$, respectively. Statistical analysis indicated that equol treatment significantly improved the mean survival of $C$. elegans under juglone-induced oxidative stress $(P<0.01)$.

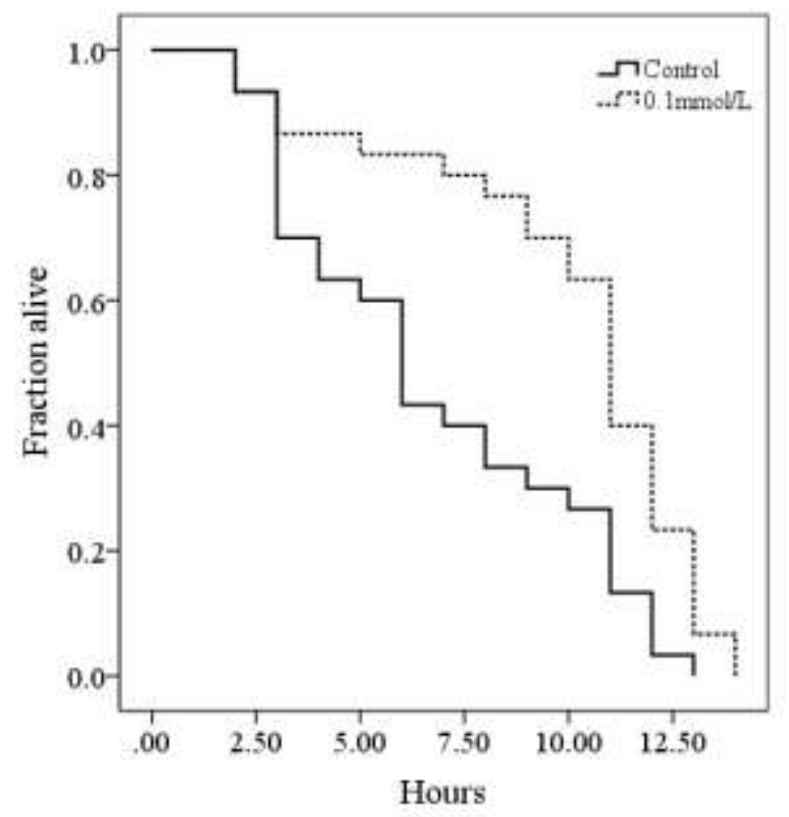

Fig.4 Effect of equol on resistance against oxidative stress of C. elegans caused by juglone

\section{Conclusions}

Our study demonstrated that equol not only significantly increased the lifespan of $C$. elegans but also significantly enhanced the resistance against both thermal and oxidative stress at the concentration of $0.1 \mathrm{mmol} / \mathrm{L}$. Neither daidzein nor DHD influenced the lifespan of C. elegans; however, $O$-Dma significantly shortened the lifespan of $C$. elegans at the concentration of 0.1 $\mathrm{mmol} / \mathrm{L}$.

\section{Acknowledgement}

Foundation items: This research was financially supported by Applied and Fundamental Research Plan in Hebei Province of China: Key Basic Research Project ( Grant No.16962504D)

\section{References}

[1] C.E. Rüfer, S.E. Kulling, Antioxidant activity of isoflavones and their major metabolites using different in vitro assays, J. Agri. Food Chem. 54 (2006) 2926-2931.

[2] S. Widyarini, A.J. Husband, V.E. Reeve, Protective effect of the isoflavonoid equol against hairless mouse skin carcinogenesis induced by UV radiation alone or with a chemical cocarcinogen, Photochem. Photobiol. 81 (2005) 32-37.

[3] S. Kreïkamp-Kaspers, L. Kok, M.L. Bots, G.D.E. robbee, J.W. Lampe, Y.T. van der Schouw, Randomized controlled trial of the effects of soy protein containing isoflavones on vascular function in postmenopausal women, Am. J. Clin. Nutr. 81 (2005) 189-195. 
[4] S. Levis, N. Strickman-Stein, P. Ganjei-Azar, P. Xu, D.R. Doerge, J. Krischer, Soy isoflavones in the prevention of menopausal bone loss and menopausal symptoms, Arch. Intern. Med. 171 (2011) 1363-1369.

[5] T.E. Hedlund, P.D. Maroni, P.G. Ferucci, R. Dayton, S. Barnes, K. Jones, R. Moore, L.G. Ogden, K. Wähälä, H.M. Sackett, K.J. Gray, Long-term dietary habits affect soy isoflavone metabolism and accumulation in prostatic fluid in caucasian men, J. Nutr. 135 (2005) 1400-1406.

[6] M. Uehara, Isoflavone metabolism and bone-sparing effects of daidzein-metabolites, J. Clin. Biochem. Nutr. 52(2013) 193-201.

[7] T.E. Hedlund, W.U. Johannes, G.J. Miller, Soy isoflavonoid equol modulates the growth of benign and malignant prostatic epithelial cells in vitro, Prostate. 54 (2003) 68-78.

[8] X.L. Liang, X.L. Wang, Z. Li, Q.H. Hao, S.Y. Wang, Improved in vitro assays of superoxide anion and 1,1-diphenyl-2-picrylhydrazyl (DPPH) radical-scavenging activity of isoflavones and isoflavone metabolites, J. Agric. Food Chem., 58 (2010) 11548-11552.

[9] D.L. Riddle, T. Blumenthal, B.J. Meyer, J.R. Priess (ed.), C. elegans II. Cold Spring Harbor Laboratory Press, Cold Spring Harbor, New York, 1997.

[10] E. Kimberley, J.C. James, H. Cheng, H. Stacie, K. Kerry, Valproic acid extends Caenorhabditis elegans lifespan, Aging Cell. 7 (2008) 305-317.

[11] H. Zhao, X.L. Wang, H.L. Zhang, C.D. Li, S.Y. Wang. Production of dihydrodaidzein and dihydrogenistein by a novel oxygen-tolerant bovine rumen bacterium in the presence of atmospheric oxygen, Appl. Microbiol. Biotechnol., 92 (2011) 803-813.

[12] X.L. Wang, J.Z. Shao, S.Y. Wang, F. Yu, Q. Zhang, C.D. Li, Chinese patent ZL 200810147314.9 (2011)

[13] M. Li, C.H. Zhang, X.L. Wang, B.H. Chen, Q.H. Hao, S.Y. Wang, Enhanced biosynthesis of $\mathrm{O}$-desmethylangolensin from daidzein by a novel oxygen-tolerant cock intestinal bacterium in the presence of atmospheric oxygen, J. Appl. Microbiol. 118 (2015) 619-628.

[14] S. Brenner, The genetics of Caenorhabditis elegans, Genetics. 77 (1974) 71-94. 